\title{
Asymptotic-sequentially solution style for the generalized Caputo time-fractional Newell-Whitehead-Segel system
}

\author{
Mohammed $\mathrm{Ali}^{1 *}$, Marwan Alquran ${ }^{1}$ and Imad Jaradat ${ }^{1}$
}

"Correspondence: myali@just.edu.jo 1 Department of Mathematics and Statistics, Jordan University of Science and Technology, Irbid, Jordan

\section{Springer}

\begin{abstract}
The Caputo fractional version of the generalized Newell-Whitehead-Segel model is considered. We introduced a numerical scheme to solve analytically the proposed application. We updated the style of the generalized Taylor series for a reliable treatment of the time-fractional derivative. The effect of the fractional derivative is explored on the obtained solutions for different cases of the problem. A sequential-asymptotic phenomenon has been observed upon varying the order of the fractional derivative from no-memory " $\alpha=0$ " to full-memory " $\alpha=1$ ".
\end{abstract}

MSC: 26A33; 34A25; 35R11

Keywords: Caputo fractional derivative; Generalized Newell-Whitehead-Segel model; Fractional-power series

\section{Introduction}

Nonlinear physical models with involved time-fractional derivative exhibited an oscillatory or chaotic or pattern states. These states occur due to a change in the order of the fractional order varying from 0 to 1 . It has been stated in [1] that most of the dynamicalphysical models with involved fractional derivatives may possess kind of hereditary features, processes or memory. In this work, we aim to explore some of the aforementioned aspects of the fractional derivative if it is considered instead of the integer derivative. To achieve our goals, we consider the time-fractional version of Newell-Whitehead-Segel (NWS) [2-5], which reads

$$
\mathcal{D}_{t}^{\alpha}[w(x, t)]=a w_{x x}(x, t)+b w(x, t)-c w^{m}(x, t),
$$

with $\alpha \in(0,1]$ being the Caputo fractional derivative where $a \in \mathbb{R}^{+}, b, c \in \mathbb{R}, m \in \mathbb{N}, x \in \mathbb{R}$, and $t \in \mathbb{R}_{>0}$.

When $\alpha=1$, the standard NWS is a $(1+1)$-dimensional amplitude system arises in fluid dynamics and describes the appearance of the stripe pattern and amplitude modulation $[6,7]$. The fractional NWS equation given in (1.1) with $\alpha$ being the Jumarie derivative has been considered in [8] and the Jumarie fractional complex transform combined with He's

(c) The Author(s) 2019. This article is distributed under the terms of the Creative Commons Attribution 4.0 International License (http://creativecommons.org/licenses/by/4.0/), which permits unrestricted use, distribution, and reproduction in any medium, provided you give appropriate credit to the original author(s) and the source, provide a link to the Creative Commons license, and indicate if changes were made. 
polynomials method is used to obtain fractional-soliton solutions. In [9], the homotopy perturbation method combined with separating variables method is used to find approximate solutions in Caputo sense. Recently, the fractional variational iteration method [10] was used to solve (1.1). In the current work, a modified fractional-power series scheme [11-16] will be used to solve analytically the fractional NWS.

We should point here that many effective methods have been developed and used to study fractional models. Worth to mention, new looks of the fractional-power series method have been recently invested to solve different types of time-space physical models [17-20], and the reproducing kernel Hilbert-space method has been used to solve many fractional-physical applications [21-26].

The paper is organized as follows: The numerical scheme to solve the time-fractional NWS is established in Sect. 2. Three different cases of NWS are explored in Sect. 3. Finally, we summarized the findings of the current work in Sect. 4 .

\section{Analysis of the proposed method}

Here, we offer a comparable version of the generalized fractional Taylor power series in which the coefficients of the series solution are obtained by the Caputo fractional differentiation instead of equating the coefficients of the same like terms. The method is particularly utilized to provide an analytical solution of Caputo time-fractional NewellWhitehead-Segel initial value problem which reads

$$
\begin{aligned}
& \mathcal{D}_{t}^{\alpha}[w(x, t)]=a w_{x x}(x, t)+b w(x, t)-c w^{m}(x, t), \\
& w(x, 0)=\xi(x),
\end{aligned}
$$

where $\alpha \in(0,1]$ is the Caputo fractional-derivative order with $a \in \mathbb{R}^{+}, b, c \in \mathbb{R}, m \in \mathbb{N}$, $x \in \mathbb{R}$, and $t \in \mathbb{R}_{\geq 0}$. The proposed solution of the problem (2.1) is written as a fractionalpower series of the form

$$
w(x, t)=\sum_{k=0}^{\infty} \xi_{k}(x) t^{k \alpha}
$$

where $\xi_{k}(x)$ are real-valued coefficient functions. Since our methods depends completely on the Caputo fractional differentiation of the power function, we recollect the fact that

$$
\mathcal{D}_{t}^{\alpha}\left[t^{\beta}\right]= \begin{cases}\frac{\Gamma(\beta+1)}{\Gamma(\beta-\alpha+1)} t^{\beta-\alpha}, & \beta>0, \\ 0, & \beta=0,\end{cases}
$$

where $\Gamma(\cdot)$ is the gamma function. Therefore, by applying the term-by-term Caputo differentiation and shifting the index we obtain the formula

$$
\mathcal{D}_{t}^{\alpha}[w(x, t)]=\sum_{k=0}^{\infty} \xi_{k+1}(x) \frac{\Gamma((k+1) \alpha+1)}{\Gamma(k \alpha+1)} t^{k \alpha} .
$$


Next, we plug both (2.2) and (2.4) into (2.1) to attain

$$
\begin{aligned}
& \sum_{k=0}^{\infty} \xi_{k+1}(x) \frac{\Gamma((k+1) \alpha+1)}{\Gamma(k \alpha+1)} t^{k \alpha} \\
& \quad=\sum_{k=0}^{\infty} a \xi_{k}^{\prime \prime}(x) t^{k \alpha}+\sum_{k=0}^{\infty} b \xi_{k}(x) t^{k \alpha}-c\left(\sum_{k=0}^{\infty} \xi_{k}(x) t^{k \alpha}\right)^{m} .
\end{aligned}
$$

In (2.5), it is clear that the function $\xi_{n}(x)$ is the coefficient of the term $t^{(n-1) \alpha}$. So to determine $\xi_{n}(x)$, it is convenient to solve the equation

$$
\left.\mathcal{D}_{t}^{(n-1) \alpha}[\boldsymbol{L}(x, t ; \alpha, n)]\right|_{t=0}=0
$$

where

$$
\begin{aligned}
\mathbf{L}(x, t ; \alpha, n)= & \sum_{k=0}^{n-1} \frac{\Gamma((k+1) \alpha+1)}{\Gamma(k \alpha+1)} \xi_{k+1}(x) t^{k \alpha}-\sum_{k=0}^{n-1} a \xi_{k}^{\prime \prime}(x) t^{k \alpha} \\
& -\sum_{k=0}^{n-1} b \xi_{k}(x) t^{k \alpha}+c\left(\sum_{k=0}^{n-1} \xi_{k}(x) t^{k \alpha}\right)^{m} .
\end{aligned}
$$

Now, we derive the first few terms of the sequence $\left\{\xi_{k}(x)\right\}_{k=1}^{n}$ where, clearly, $\xi_{0}(x)=\xi(x)$.

Step 1 . We begin with $n=1$. Thus

$$
\mathbf{L}(x, t ; \alpha, 1)=\Gamma(\alpha+1) \xi_{1}(x)-a \xi_{0}^{\prime \prime}(x)-b \xi_{0}(x)+c \xi_{0}^{m}(x) .
$$

Solving $\mathbf{L}(x, t ; \alpha, 1)=0$ with respect to $\xi_{1}(x)$ yields

$$
\xi_{1}(x)=\frac{1}{\Gamma(\alpha+1)}\left(a \xi_{0}^{\prime \prime}(x)+b \xi_{0}(x)-c \xi_{0}^{m}(x)\right)
$$

Step 2. For $n=2$, we have

$$
\mathbf{L}(x, t ; \alpha, 2)=\frac{\Gamma(2 \alpha+1)}{\Gamma(\alpha+1)} \xi_{2}(x) t^{\alpha}-a \xi_{1}^{\prime \prime}(x) t^{\alpha}-b \xi_{1}(x) t^{\alpha}+c m \xi_{0}^{m-1}(x) \xi_{1}(x) t^{\alpha}
$$

Solving $\mathcal{D}_{t}^{\alpha}[\boldsymbol{L}(x, t ; \alpha, 2)]=0$ with respect to $\xi_{2}(x)$, gives

$$
\xi_{2}(x)=\frac{\Gamma(\alpha+1)}{\Gamma(2 \alpha+1)}\left(a \xi_{1}^{\prime \prime}(x)+b \xi_{1}(x)-c m \xi_{0}^{m-1}(x) \xi_{1}(x)\right) .
$$

Remark As the chain rule differentiation is not valid in Caputo sense, we have to expand all terms in $L(x, t ; \alpha, n)$ and then use the fact that

$$
\left.\mathcal{D}_{t}^{n \alpha}\left[t^{\beta}\right]\right|_{t=0}= \begin{cases}\Gamma(n \alpha+1), & \beta=n \alpha \\ 0, & \text { otherwise }\end{cases}
$$


Step 3. To find $\xi_{3}(x)$, we consider $\boldsymbol{L}(x, t ; \alpha, 3)$ and then we solve $\left.\mathcal{D}_{t}^{2 \alpha}[\boldsymbol{L}(x, t ; \alpha, 3)]\right|_{t=0}=0$. Hence

$$
\begin{aligned}
\xi_{3}(x)= & \frac{\Gamma(2 \alpha+1)}{\Gamma(3 \alpha+1)}\left(a \xi_{2}^{\prime \prime}(x)+b \xi_{2}(x)-c\left(\left(\begin{array}{c}
m \\
2
\end{array}\right) \xi_{0}^{m-2}(x) \xi_{1}^{2}(x)\right.\right. \\
& \left.\left.+\left(\begin{array}{c}
m \\
1
\end{array}\right) \xi_{0}^{m-1}(x) \xi_{2}(x)\right)\right) .
\end{aligned}
$$

Step 4 . To find $\xi_{4}(x)$, we consider $\boldsymbol{L}(x, t ; \alpha, 4)$ and then we solve $\left.\mathcal{D}_{t}^{3 \alpha}[\boldsymbol{L}(x, t ; \alpha, 4)]\right|_{t=0}=0$. Therefore

$$
\begin{aligned}
\xi_{4}(x)= & \frac{\Gamma(3 \alpha+1)}{\Gamma(4 \alpha+1)}\left(a \xi_{3}^{\prime \prime}(x)+b \xi_{3}(x)-c\left(\left(\begin{array}{c}
m \\
3
\end{array}\right) \xi_{0}^{m-3}(x) \xi_{1}^{3}(x)\right.\right. \\
& \left.\left.+2\left(\begin{array}{c}
m \\
2
\end{array}\right) \xi_{0}^{m-2}(x) \xi_{1}(x) \xi_{2}(x)+\left(\begin{array}{c}
m \\
1
\end{array}\right) \xi_{0}^{m-1}(x) \xi_{3}(x)\right)\right) .
\end{aligned}
$$

Finally, by proceeding in the same manner, solving $\left.\mathcal{D}_{t}^{(k-1) \alpha}[\ell(x, t ; \alpha, k)]\right|_{t=0}=0$ yields

$$
\xi_{k}(x)=\frac{\Gamma((k-1) \alpha+1)}{\Gamma(k \alpha+1)}\left(a \xi_{k-1}^{\prime \prime}(x)+b \xi_{k-1}(x)-c \Upsilon_{k}(x)\right)
$$

where

$$
\begin{aligned}
& \Upsilon_{0}(x)=\xi_{0}^{m}(x), \\
& \Upsilon_{k}(x)=\frac{1}{k \xi_{0}(x)} \sum_{i=1}^{k}(i m-k+i) \xi_{i}(x) \Upsilon_{k-i}(x)
\end{aligned}
$$

It is worth mentioning here that the solution of the integer-version of Newell-WhiteheadSegel initial value problem has been achieved using the homotopy perturbation method (HPM) in [5]. In fact, four study cases have been discussed there. By a straightforward substitution, one verifies that all their obtained solutions agree with our general formulas (2.15)-(2.16) by just letting the fractional derivative $\alpha$ approaches one. Therefore, their adopted numerical trend to show the rapid convergence toward the exact solution is valid in our case when our study is projected to the integer space. This shows the generality of the fractional derivatives.

For comparison purposes, a recursive formula for all the series coefficients is obtained in our approach while a few terms are obtained using HPM. Further, the HPM completely depends on the choice of an appropriate auxiliary operator and an initial guess for the solution. While our approach merely assumed the existence of the solution as a fractionalpower series.

\section{Applications and discussion}

In this section, we apply the previous procedure for three study cases to test the validity of our suggested solution. 
Case 1 Consider the following time-fractional Newell-Whitehead-Segel initial value problem:

$$
\left\{\begin{array}{l}
\mathcal{D}_{t}^{\alpha}[w(x, t)]=5 w_{x x}(x, t)+2 w(x, t)+w^{2}(x, t) \\
u(x, 0)=\kappa \in \mathbb{R}
\end{array}\right.
$$

Using the previous procedure, we get the following first few coefficients:

$$
\begin{aligned}
& \xi_{1}(x)=\frac{\kappa(\kappa+2)}{\Gamma(\alpha+1)}, \\
& \xi_{2}(x)=\frac{2 \kappa\left(\kappa^{2}+3 \kappa+2\right)}{\Gamma(2 \alpha+1)}, \\
& \xi_{3}(x)=\frac{\kappa(\kappa+2)\left(4(\kappa+1)^{2} \Gamma(\alpha+1)^{2}+\kappa(\kappa+2) \Gamma(2 \alpha+1)\right)}{\Gamma(\alpha+1)^{2} \Gamma(3 \alpha+1)}, \\
& \xi_{4}(x)=\frac{2 \kappa\left(\kappa^{2}+3 \kappa+2\right)\left(4(\kappa+1)^{2} \Gamma(2 \alpha+1) \Gamma(\alpha+1)^{2}+2 \kappa(\kappa+2) \Gamma(3 \alpha+1) \Gamma(\alpha+1)+\kappa(\kappa+2) \Gamma(2 \alpha+1)^{2}\right)}{\Gamma(\alpha+1)^{2} \Gamma(2 \alpha+1) \Gamma(4 \alpha+1)} .
\end{aligned}
$$

Case 2 Consider the following time-fractional Newell-Whitehead-Segel initial value problem:

$$
\begin{aligned}
& \mathcal{D}_{t}^{\alpha}[w(x, t)]=w_{x x}(x, t)+2 w(x, t)-3 w^{2}(x, t), \\
& u(x, 0)=\kappa \in \mathbb{R} .
\end{aligned}
$$

Using the previous procedure, we get the following first few coefficients:

$$
\begin{aligned}
& \xi_{1}(x)=\frac{\kappa(2-3 \kappa)}{\Gamma(\alpha+1)} \\
& \xi_{2}(x)=\frac{2 \kappa\left(9 \kappa^{2}-9 \kappa+2\right)}{\Gamma(2 \alpha+1)} \\
& \xi_{3}(x)=-\frac{\kappa(3 \kappa-2)\left(4(1-3 \kappa)^{2} \Gamma(\alpha+1)^{2}+3 \kappa(3 \kappa-2) \Gamma(2 \alpha+1)\right)}{\Gamma(\alpha+1)^{2} \Gamma(3 \alpha+1)} \\
& \xi_{4}(x)=\frac{2 \kappa\left(9 \kappa^{2}-9 \kappa+2\right)\left(4(1-3 \kappa)^{2} \Gamma(2 \alpha+1) \Gamma(\alpha+1)^{2}+6 \kappa(3 \kappa-2) \Gamma(3 \alpha+1) \Gamma(\alpha+1)+3 \kappa(3 \kappa-2) \Gamma(2 \alpha+1)^{2}\right)}{\Gamma(\alpha+1)^{2} \Gamma(2 \alpha+1) \Gamma(4 \alpha+1)} .
\end{aligned}
$$

Case 3 Consider the following time-fractional Newell-Whitehead-Segel initial value problem:

$$
\begin{aligned}
& \mathcal{D}_{t}^{\alpha}[w(x, t)]=w_{x x}(x, t)+2 w(x, t)-3 w^{3}(x, t), \\
& u(x, 0)=\sqrt{\frac{2}{3}} \frac{e^{x}}{1+e^{x}} .
\end{aligned}
$$




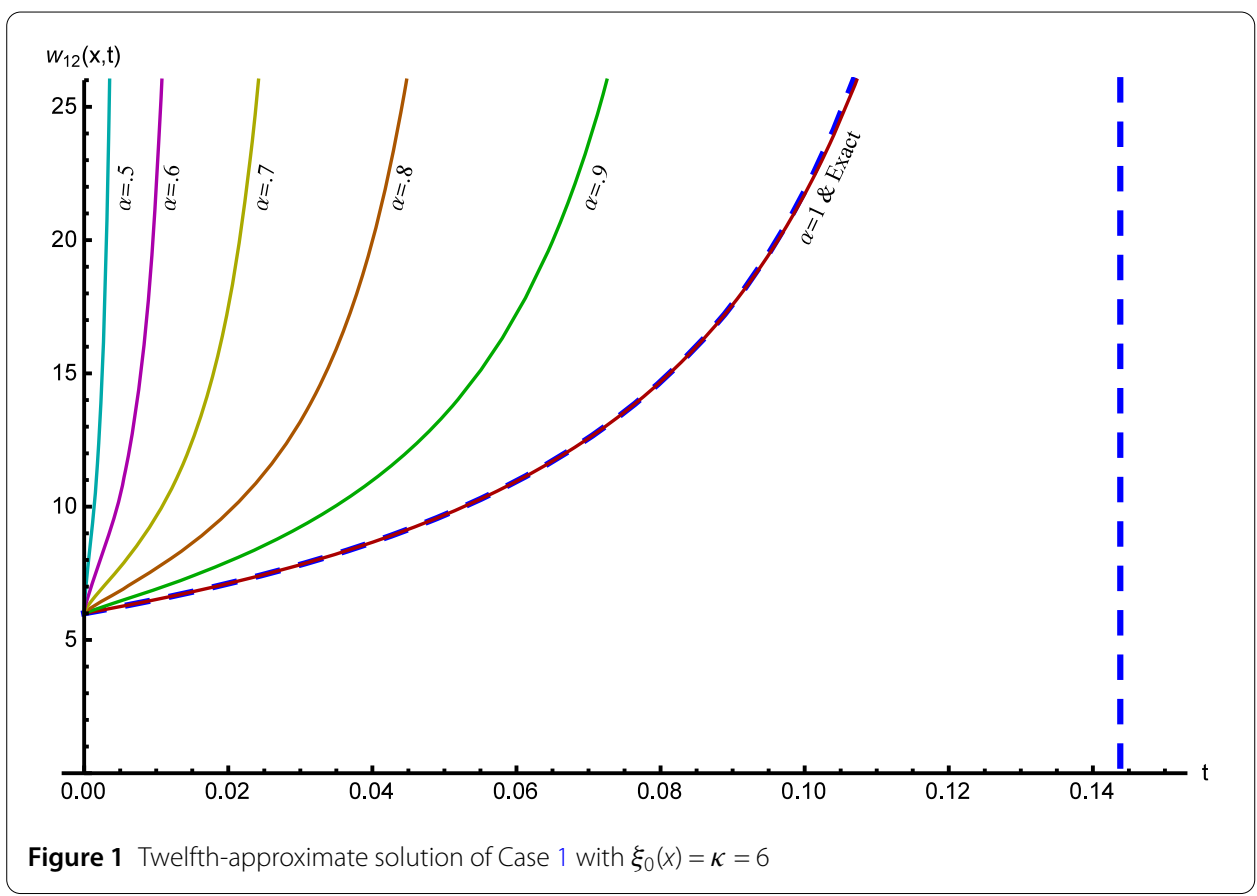

Using the previous procedure, we get the following first few coefficients:

$$
\begin{aligned}
\xi_{1}(x)= & \frac{\sqrt{6} e^{x}}{\left(e^{x}+1\right)^{2} \Gamma(\alpha+1)}, \\
\xi_{2}(x)= & -\frac{3 \sqrt{6} e^{x}\left(e^{x}-1\right)}{\left(e^{x}+1\right)^{3} \Gamma(2 \alpha+1)}, \\
\xi_{3}(x)= & \frac{9 \sqrt{6} e^{x}\left(\left(-3 e^{x}+e^{2 x}+e^{3 x}+1\right) \Gamma(\alpha+1)^{2}-2 e^{2 x} \Gamma(2 \alpha+1)\right)}{\left(e^{x}+1\right)^{5} \Gamma(\alpha+1)^{2} \Gamma(3 \alpha+1)}, \\
\xi_{4}(x)= & -9 \sqrt{6} e^{x}\left(\frac{\left(27 e^{x}-62 e^{2 x}+10 e^{3 x}+9 e^{4 x}+3 e^{5 x}-3\right) \Gamma(2 \alpha+1) \Gamma(\alpha+1)^{3}}{\left(e^{x}+1\right)^{7} \Gamma(\alpha+1)^{3} \Gamma(2 \alpha+1) \Gamma(4 \alpha+1)}\right. \\
& \left.-\frac{12 e^{2 x}\left(e^{2 x}-1\right) \Gamma(3 \alpha+1) \Gamma(\alpha+1)^{2}+2 e^{2 x}\left(13 e^{x}-11 \Gamma(2 \alpha+1)^{2} \Gamma(\alpha+1)-2 e^{2 x}\left(e^{x}+1\right) \Gamma(2 \alpha+1) \Gamma(3 \alpha+1)\right)}{\left(e^{x}+1\right)^{7} \Gamma(\alpha+1)^{3} \Gamma(2 \alpha+1) \Gamma(4 \alpha+1)}\right) .
\end{aligned}
$$

Figures 1, 2, and 3 exhibit the behavior of the approximate solutions for Cases 1, 2, and 3, respectively, labeled with various values of the fractional-derivative parameter $\alpha \in(0,1)$. Evidently, the graphs show an asymptotic-sequentially solution style when $\alpha$ varies from 0 "no-memory" to 1 "full-memory".

\section{Conclusions}

A comparable version of the generalized fractional Taylor power series is introduced to find analytical solutions of the generalized time-fractional Newell-Whitehead-Segel equation. Different cases of the model are tested by the proposed modified method. The effect of the embedded Caputo fractional derivative provided an asymptotic-sequentially solution style when $\alpha$ varies from 0 "no-memory" to 1 "full-memory."

The advantages achieved by using the modified fractional-power series scheme can be tagged as follows:

- It can be utilized regardless of the chosen type of the fractional derivative since almost all the fractional-types agree with the rule of the power function differentiation. 

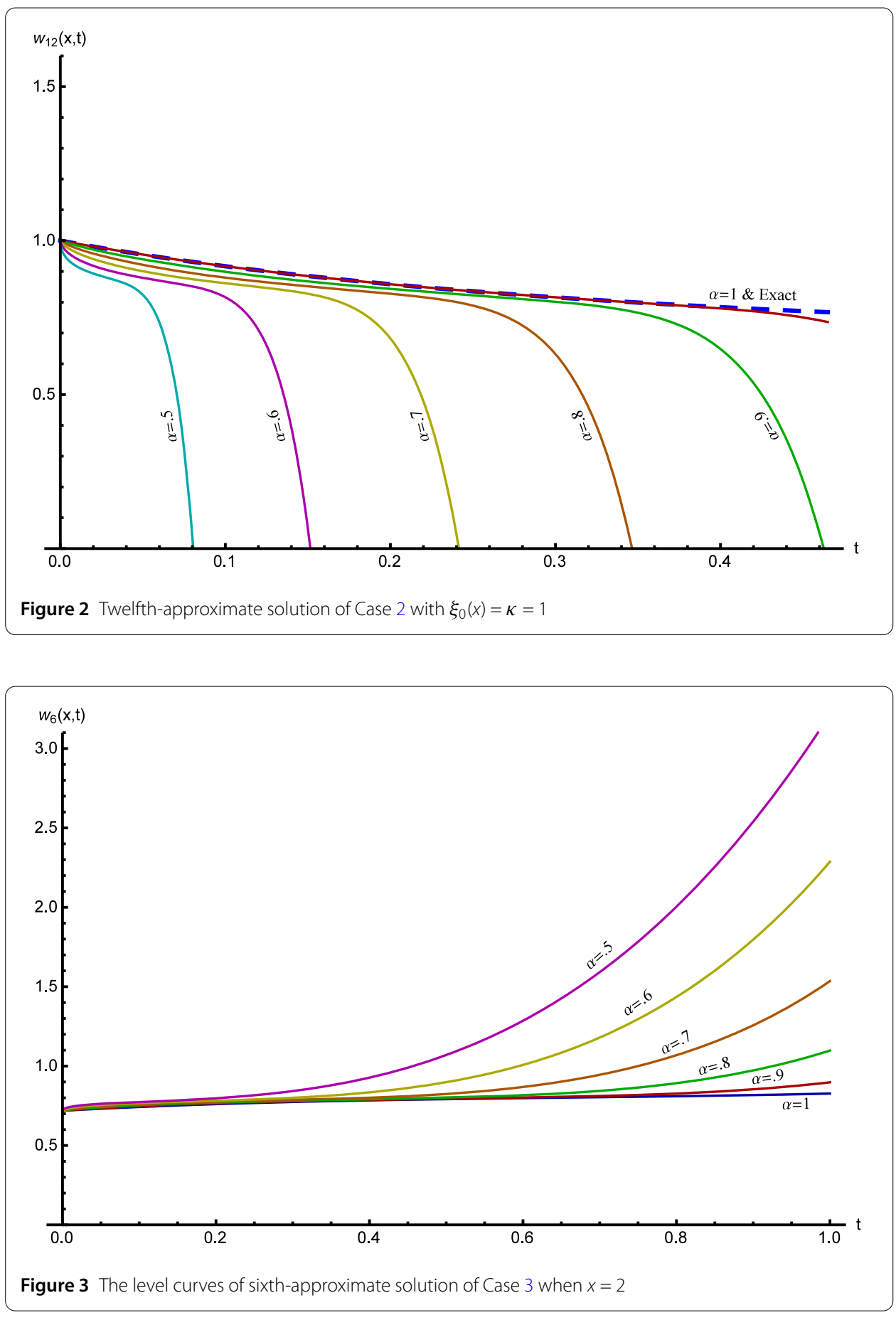

- It requires less amount of computational work with convenient accuracy.

- It provides a delicate iterative scheme in determining the solutions components with fast execution-time.

- The obtained fractional-power-series solution is stable while the values of the fractional order $\alpha$ is approaching 1.

Accordingly, as future work, the proposed method can be extended to investigate more fractional-physical models. 


\section{Acknowledgements}

Authors would like to thank the editor and the anonymous referees for their in-depth reading and insightful comments on an earlier version of this paper.

\section{Funding}

Not applicable.

\section{Availability of data and materials \\ Not applicable.}

\section{Ethics approval and consent to participate}

Not applicable.

\section{Competing interests}

The authors declare that there is no conflict of interests regarding the publication of this manuscript. The authors declare that they have no competing interests.

\section{Consent for publication}

Not applicable.

\section{Authors' contributions}

All authors contributed equally and read and approved the final version of the manuscript.

\section{Publisher's Note}

Springer Nature remains neutral with regard to jurisdictional claims in published maps and institutional affiliations.

Received: 19 November 2018 Accepted: 12 February 2019 Published online: 21 February 2019

\section{References}

1. Caputo, M., Mainardi, F.: A new dissipation model based on memory mechanism. Pure Appl. Geophys. 91 (1971)

2. Saravanan, A., Magesh, N.: A comparison between the reduced differential transform method and the Adomian decomposition method for the Newell-Whitehead-Segel equation. J. Egypt. Math. Soc. 21(3), 259-265 (2013)

3. Patade, J., Bhalekar, S.: Approximate analytical solutions of Newell-Whitehead-Segel equation using a new iterative method. World J. Model. Simul. 11(2), 94-103 (2015)

4. Prakash, A., Kumar, M.: He's variational iteration method for the solution of nonlinear Newell-Whitehead-Segel equation. J. Appl. Anal. Comput. 6(3), 738-748 (2016)

5. Nourazar, S.S., Soori, M., Nazari-Golshan, A.: On the exact solution of Newell-Whitehead-Segel equation using the homotopy perturbation method. Aust. J. Basic Appl. Sci. 5(8), 1400-1411 (2011)

6. Newell, A., Whitehead, J.: Finite bandwidth, finite amplitude convection. J. Fluid Mech. 38(2), 279-303 (1969)

7. Segel, L.: Distant side-walls cause slow amplitude modulation of cellular convection. J. Fluid Mech. 38(1), 203-224 (1969)

8. Edeki, S.O., Ejiogu, J.I., Ejoh, S.A., Adeyemi, G.A.: Coupled FCT-HP for analytical solutions of the generalized time-fractional Newell-Whitehead-Segel equation. Int. J. Pure Math. 5, 29-32 (2018)

9. Bagherpur, H., Kheri, H., Mojaver, A.: The analytical solutions of the tine-fractional Newell-Whitehead-Segel equation by the method of modified homotopy perturbation and separating variables. Adv. Stud. Contemp. Math. 24(4), 499-514 (2014)

10. Prakash, A., Goyal, M., Gupta, S.: Fractional variational iteration method for solving time-fractional Newell-Whitehead-Segel equation. Nonlinear Eng. 5(2), 81-86 (2018)

11. Alquran, M., Jaradat, I.: A novel scheme for solving Caputo time-fractional nonlinear equations: theory and application. Nonlinear Dyn. 91(4), 2389-2395 (2018)

12. El-Ajou, A., Abu-Arqub, O., Al-Zhour, Z., Momani, S.: New results on fractional power series: theories and applications. Entropy 15, 5305-5323 (2013)

13. Jaradat, H.M., Al-Shara, S., Khan, Q.J.A., Alquran, M., Al-Khaled, K.: Analytical solution of time-fractional Drinfeld-Sokolov-Wilson system using residual power series method. IAENG Int. J. Appl. Math. 46(1), 64-70 (2016)

14. Jaradat, H.M., Jaradat, I., Alquran, M., Jaradat, M.M.M., Mustafa, Z., Abohassan, K., Abdelkarim, R.: Approximate solutions to the generalized time-fractional Ito system. Ital. J. Pure Appl. Math. 37, 699-710 (2017)

15. Alquran, M., Al-Khaled, K., Sivasundaram, S., Jaradat, H.M.: Mathematical and numerical study of existence of bifurcations of the generalized fractional Burgers-Huxley equation. Nonlinear Stud. 24(1), 235-244 (2017)

16. Alquran, M., Jaradat, I., Sivasundaram, S.: Elegant scheme for solving Caputo-time-fractional integro-differential equations. Nonlinear Stud. 25(2), 385-393 (2018)

17. Jaradat, I., Alquran, M., Al-Khaled, K.: An analytical study of physical models with inherited temporal and spatial memory. Eur. Phys. J. Plus 133, 162 (2018)

18. Jaradat, I., Al-Dolat, M., Al-Zoubi, K., Alquran, M.: Theory and applications of a more general form for fractional power series expansion. Chaos Solitons Fractals 108, 107-110 (2018)

19. Jaradat, I., Alquran, M., Al-Dolat, M.: Analytic solution of homogeneous time-invariant fractional IVP. Adv. Differ. Equ. 2018, $143(2018)$

20. Jaradat, I., Alquran, M., Abdel-Muhsen, R.: An analytical framework of 2D diffusion, wave-like, telegraph, and Burgers models with twofold Caputo derivatives ordering. Nonlinear Dyn. 93(4), 1911-1922 (2018)

21. Abu-Arqub, O., Odibat, Z., Al-Smadi, M.: Numerical solutions of time-fractional partial integro-differential equations of Robin functions types in Hilbert space with error bounds and error estimates. Nonlinear Dyn. 94(3), 1819-1834 (2018) 
22. Al-Smadi, M., Abu-Arqub, O.: Computational algorithm for solving Fredholm time-fractional partial integro-differential equations of Dirichlet functions type with error estimates. Appl. Math. Comput. 342, 280-294 (2019)

23. Abu-Arqub, O., Al-Smadi, M.: Atangana-Baleanu fractional approach to the solutions of Bagley-Torvik and Painlevé equations in Hilbert space. Chaos Solitons Fractals 117, 161-167 (2018)

24. Abu-Arqub, O., Maayah, B.: Numerical solutions of integro-differential equations of Fredholm operator type in the sense of the Atangana-Baleanu fractional operator. Chaos Solitons Fractals 117, 117-124 (2018)

25. Abu-Arqub, O., Al-Smadi, M.: Numerical algorithm for solving time-fractional partial integro-differential equations subject to initial and Dirichlet boundary conditions. Numer. Methods Partial Differ. Equ. 34(5), 1577-1597 (2018)

26. Abu-Arqub, O.: Solutions of time-fractional Tricomi and Keldysh equations of Dirichlet functions types in Hilbert space. Numer. Methods Partial Differ. Equ. 34(5), 1759-1780 (2018)

Submit your manuscript to a SpringerOpen ${ }^{0}$ journal and benefit from:

- Convenient online submission

- Rigorous peer review

Open access: articles freely available online

- High visibility within the field

- Retaining the copyright to your article

Submit your next manuscript at $\boldsymbol{~ s p r i n g e r o p e n . c o m ~}$ 